Relations industrielles

Industrial Relations

\title{
Roger G. MARTIN : Annuaire du travail 1986-1987. Montréal, Productions INFORT, 1986
}

\section{Gérard Dion}

Volume 41, numéro 4, 1986

URI : https://id.erudit.org/iderudit/050277ar

DOI : https://doi.org/10.7202/050277ar

Aller au sommaire du numéro

Éditeur(s)

Département des relations industrielles de l'Université Laval

ISSN

0034-379X (imprimé)

1703-8138 (numérique)

Découvrir la revue

Citer ce compte rendu

Dion, G. (1986). Compte rendu de [Roger G. MARTIN : Annuaire du travail 1986-1987. Montréal, Productions INFORT, 1986]. Relations industrielles / Industrial Relations, 41(4), 886-887. https://doi.org/10.7202/050277ar

Tous droits réservés (C) Département des relations industrielles de l'Université Laval, 1986
Ce document est protégé par la loi sur le droit d'auteur. L’utilisation des services d'Érudit (y compris la reproduction) est assujettie à sa politique d'utilisation que vous pouvez consulter en ligne.

https://apropos.erudit.org/fr/usagers/politique-dutilisation/ 
La deuxième partie du document utilise un grand nombre d'exemples concrets tirés des cas étudiés pour illustrer les différentes phases de l'implantation d'un programme de cercles de qualité: (1) la décision d'adopter une approche participative et d'opter pour les cercles de qualité ou un programme semblable; (2) la planification du programme, la mise en place des structures et l'attribution des responsabilités; (3) le démarrage des premiers cercles et l'élaboration des programmes de formation nécessaires; (4) le suivi et l'évaluation du programme de même que l'évolution graduelle des structures et des rôles; (5) les efforts nécessaires pour stimuler et revigorer le programme après deux ou trois ans. Une dernière section (malheureusement très courte et très faible) examine quelques résultats obtenus et divise ceux-ci en trois catégories: l'impact sur les ressources humaines (climat, style de leadership), sur la productivité et la qualité du produit, sur les relations de travail.

La troisième partie résume l'essentiel des 11 cas qui ont fait l'objet d'un examen plus approfondi, en consacrant environ deux pages à chacun.

Ce document de 77 pages ne cherche évidemment pas à remplacer les nombreux textes de base sur les cercles de qualité. Il s'adresse surtout à ceux qui connaissent déjà «la théorie» des cercles de qualité et qui veulent savoir comment les choses se passent «en pratique». Ce qu'on y apprend, c'est que les choses se passent à peu près comme on nous l'avait prédit dans les manuels de base: il faut une volonté réelle de changer la culture et les modes de gestion, il faut suivre religieusement toutes les étapes prévues, il faut de la patience, de la détermination, du temps (environ un an) et même de la chance (pas de bouleversement économique ou social majeur pendant la longue période d'incubation).

Au delà d'une certaine uniformité dans les principes de base et les étapes d'implantation, le document démontre une grande diversité entre les entreprises en ce qui concerne la plupart des éléments du programme: les objectifs visés, l'influence réelle du syndicat, la composition et le rôle du comité directeur, les programmes de formation, les stratégies d'extension, le partage des gains de productivité, l'évaluation et la diffusion des résultats, les façons de soutenir le programme. Il faut tout adapter à la situation de chaque entreprise et c'est ce qui se fait continuellement.

Ce document de 77 pages est intéressant et instructif, mais il ne faut pas en attendre autre chose que ce qu'il prétend faire: résumer pour nous d'une façon très rapide et un peu superficielle plusieurs cas d'entreprises canadiennes qui sont passées par toutes les étapes d'un programme de cercles de qualité et en tirer quelques conclusions élémentaires. Toute réflexion profonde sur le phénomène lui-même n'a pas sa place ici (e.g. si toutes les entreprises doivent travailler si fort pour assurer la survie du programme après deux ou trois ans, le concept lui-même ne doit-il pas être repensé?).

Jean-Louis BFRGERON

Université de Sherbrooke

Annuaire du travail 1986-1987, par Roger G. Martin, Montréal, Productions INFORT, 1986.

Il s'agit de la deuxième édition d'un ouvrage publié d'abord en 1985. Il n'y a pas de pagination d'ensemble et la maison d'édition ne s'est pas souciée d'obtenir un ISBN. Sous onze rubriques, l'auteur Roger G. Martin, professeur agrégé au H.E.C. présente une nomenclature des personnes ou des institutions qui oeuvrent dans le domaine des relations du travail au Québec: arbitres; entreprises conseils; institutions d'enseignement et centres de documentation; maisons de publication; ministères; organismes paritaires, organismes patronaux, organismes professionnels; organismes syndicaux; praticiens. 
L'auteur s'est donné la peine de compiler dans un même ouvrage une multitude d'informations qu'il est allé chercher à différentes sources dont l'accessibilité n'est pas toujours facile et qui doivent constamment être mises à jour. La seconde édition, tout en conservant les mêmes rubriques, s'est enrichie de nombreux renseignements. Il faut cependant constater qu'avec la suppression de la table des matières et de la pagination, la consultation est rendue difficile. Il va falloir que l'auteur trouve un système quelconque, comme par exemple celui des Editions FM, s'il ne veut pas que les lecteurs perdent patience et délaissent cet instrument de travail.

L'auteur a fait une oeuvre utile. Il est bien conscient que l'Annuaire du travail comporte des lacunes et peut être amélioré. Il sait que son utilité restera fonction de la qualité des informations qu'il contient et de la rapidité avec laquelle l'usager peut les obtenir. Ce n'est pas toujours le cas, particulièrement sous la rubrique "entreprises conseils». La rubrique "ministères: juridiction fédérale» m'apparaît bien incomplète. On n'y trouve même pas l'adresse du CRTC à Ottawa ni le nom des commissaires parmi les praticiens. Peut-être, est-ce un choix volontaire de l'auteur? En tous cas, le lecteur saurait mieux à quoi s'en tenir s'il avait trouvé une introduction à l'ouvrage où auraient été expliqués les critères qui ont présidé au choix des insertions. Il me semble qu'une rubrique comprenant les périodiques en relations du travail aurait sa place dans un tel annuaire.

L'auteur a été courageux en entreprenant une publication de ce genre. On n'a pas à être surpris si un rodage s'impose pour qu'elle réalise l'objectif poursuivi.

Gérard DION

Université Laval

\section{LIVRES REÇUS BOOKS RECEIVED}

LIPSET, Seymour Martin Unions in Transition. Entering the Second Century, San Francisco, Institute for Contemporary Studies, 1986, 506 pp., ISBN 0-917616-74-X and ISBN 0-917616-73-1 (pbk)

INDUSTRIAL RELATIONS RESEARCH ASSOCIATION, Proceedings of the 1986 Spring Meeting, Atlanta, Georgia, April 17-18, 1986, 591 pp., ISBN 0-913447-32-3

INDUSTRIAL RELATIONS RESEARCH ASSOCIATION SERIES, Proceedings of the Thirty-Eight Annual Meeting, New York, December 28-30, 1985, 536 pp., ISBN 0-913447-31-5
DAVID, Hélène, Femmes et emploi. Le défi de l'égalité, PUQ, Sillery et IRAT, Montréal, 1986, 477 pp., ISBN 2-7605-0393-3

GILSON, Ch. J. (ed.), Strikes in Nova Scotia, Hantsport, Nova Scotia, 1986, 191 pp., ISBN 0-88999-314-9

HANNAH, Leslie, Inventing Retirement. The Development of Occupational Pensions in Britain, New York, Cambridge University Press, 1986, 201 pp., ISBN 0-521-30361-3

GEARY, Roger, Policing Industrial Disputes, 1893 to 1985 , London, Methuen \& Co. Ltd., 1986, 171 pp., ISBN 0-416-90200-6 